\title{
Final report of the 5-year clinical outcomes of the XINSORB bioresorbable sirolimus-eluting scaffold in the treatment of single de novo coronary lesions in a first-in-human study
}

\author{
Yizhe Wü, Jiasheng Yin", Jiahui Chen, Zhifeng Yao, Juying Qian, Li Shen, Lei Ge, Junbo Ge; on behalf \\ of the XINSORB first-in-human study
}

Shanghai Institute of Cardiovascular Diseases, Department of Cardiology, Zhongshan Hospital, Fudan University, Shanghai, China

Contributions: (I) Conception and design: L Ge, J Ge; (II) Administrative support: J Qian, J Ge; (III) Provision of study materials or patients: Z Yao; (IV) Collection and assembly of data: J Chen; (V) Data analysis and interpretation: Y Wu, J Yin, L Shen; (VI) Manuscript writing: All authors; (VII) Final approval of manuscript: All authors.

"These authors contributed equally to this work.

Correspondence to: Junbo Ge; Lei Ge; Li Shen. 180 Fenglin Road, Shanghai, China. Email: ge.junbo2@zs-hospital.sh.cn;

ge.lei@zs-hospital.sh.cn; nt_sunny@163.com.

\begin{abstract}
Background: We aimed to report the 5-year outcomes of XINSORB bioresorbable sirolimus-eluting scaffolds in the treatment of single de novo coronary lesions in a first-in-human (FIM) study. This is the final report of the long-term clinical outcomes of the study. Recent studies have shown that bioresorbable scaffolds (BRSs) increase the risks of late target lesion failure (TLF) and thrombosis.

Methods: In this prospective, single-arm study, eligible patients with single de novo coronary lesions were enrolled and treated with XINSORB scaffolds. The scaffolds measured $3.0 \mathrm{~mm}$ in diameter and 12, 15, and $18 \mathrm{~mm}$ in length. The clinical endpoints included TLF [cardiac death, target vessel-related myocardial infarction (TV-MI), or ischaemia-driven target lesion revascularization (ID-TLR)], its components, major adverse cardiac events (MACE), and scaffold thrombosis.

Results: From September 2013 to January 2014, 30 patients were enrolled and treated with XINSORB scaffolds. The procedure had a 100\% success rate. None of the patients died during the 5 years of follow-up. The primary endpoint of TLF occurred in 4 patients (13.3\%). Six patients were recanalized by intervention, including 4 by ID-TLR. The rate of MACE was 16.7\% (5/30). One very late case of scaffold thrombosis was recorded, which led to TV-MI. No more cases of thrombosis were recorded beyond 2 years of follow-up. The rates of clinical endpoints remained steady with no changes after 3 years of follow-up.

Conclusions: Considering that this FIM study was launched at an early stage of the BRS era and without optimal implantation techniques, the clinical outcomes of TLF during the 5-year follow-up were acceptable. The rate of thrombosis was relatively low.
\end{abstract}

Keywords: Coronary artery disease; percutaneous coronary intervention (PCI); bioresorbable scaffold (BRS)

Submitted Jul 10, 2020. Accepted for publication Sep 02, 2020.

doi: $10.21037 / \mathrm{atm}-20-5668$

View this article at: http://dx.doi.org/10.21037/atm-20-5668

\section{Introduction}

Despite the considerable achievements that have been made with the use of bioresorbable scaffolds (BRSs) in the last decade, results reported recently have raised concerns regarding the long-term risks of target lesion failure (TLF) and thrombosis (1-3). However, a recently published metaanalysis indicated that this device had favourable rates of TLF and thrombosis beyond 3 years after the index procedure (4). Compared to Absorb BRS, XINSORB BRS (Shandong Huaan, China) is a contemporary scaffold. 
The first clinical trial regarding to BRS in China was the XINSORB first-in-human (FIM) study. The FIM study on XINSORB BRS commenced in September 2013. The 6-month angiographic and clinical results of this study were reported previously (5). The in-segment late luminal loss (LLL) was $0.13 \pm 0.24 \mathrm{~mm}$. No clinical events occurred during the 6-month follow-up. The randomized control trial (RCT) and registry trial of XINSORB BRS were carried out in September 2014. The results of the 1- and 3 -year follow-ups have been confirmed and were promising compared with those of a commercialized metallic sirolimus-eluting stent (6). Consequently, the XINSORB BRS was approved for use in the Chinese market by the China Food and Drug Administration (CFDA) in March 2020. Here, we present our latest analysis of the 5-year clinical outcomes of the XINSORB FIM study. This is the final report of this study.

We present the following article in accordance with the TREND reporting checklist (available at http://dx.doi. org/10.21037/atm-20-5668).

\section{Methods}

\section{Study device}

XINSORB BRS is composed of poly-L-lactic acid (PLLA). The scaffold's design is similar to that of the Absorb scaffold. The struts are coated with poly-D-L-lactic acid (PDLLA) mixed with PLLA carrying sirolimus. The dose of sirolimus ranges from $8-16 \mu \mathrm{g} / \mathrm{mm}$, depending on the length of the scaffold. The struts are $160 \mu \mathrm{m}$ in thickness. The XINSORB scaffolds used in this study measured $3.0 \mathrm{~mm}$ in diameter and 12,15 , and $18 \mathrm{~mm}$ in length.

\section{Study design, patient population, intervention, and follow-up}

This FIM study was a single-arm, prospective clinical trial. The study complied with the CFDA's Good Clinical Practice guidelines. All procedures performed in this study involving human participants were in accordance with the Declaration of Helsinki (as revised in 2013). The study was approved by institutional ethics committee of Zhongshan Hospital, Fudan University (No. 2013-36) and informed consent was taken from all the patients. The study was performed in two centres in China. The protocol was described previously (5).

Briefly, patients were eligible if they were aged between
18-75 years old and diagnosed with stable or unstable ischaemic coronary disease or subacute myocardial infarction (beyond 7 days with normal troponin, and creatine kinase and its MB isoenzyme). Single, de novo lesions in a native coronary artery were included and was visually assessed to be $<14 \mathrm{~mm}$ in length. The reference vessel diameter of the target lesion was $3.0 \mathrm{~mm}$, and the percentage of the diameter was $50-99 \%$, with a thrombolysis in myocardial infarction (TIMI) flow grade of $>1$.

The procedure was performed as standard. Predilation was mandatory, while postdilation was recommended. If postdilation was necessary, the diameter of the noncompliance balloon needed to be shorter than the implanted scaffold and up to a maximum of $0.25 \mathrm{~mm}$ larger. If bailout stenting was required, commercialized metallic drug-eluting stents were used.

All patients received regular clinical follow-up examinations at 30, 90, 180, and 270 days, and at 1, 2, 3, 4 , and 5 years. At 180 days after the index procedure, the patients underwent angiography according to the protocol. Follow-up examinations up to 5 years were completed at the time of the current report. The patients were prescribed clopidogrel for at least 12 months, and continued taking aspirin for the duration of the study.

\section{Endpoints and definitions}

The primary endpoint of the FIM study was angiographic in-segment LLL at the 6-month follow-up, which was defined as the difference in the minimal luminal diameter from postprocedure to 6 months. Restenosis was defined as $\geq 50 \%$ diameter stenosis in every stented segment and peri-scaffold segment. Device success was defined as the successful delivery and deployment of the study device at the intended target lesion to obtain a final residual stenosis of $<50 \%$, as measured by quantitative coronary angiography. Bailout stenting was not regarded as a failure of the device. Other secondary endpoints included TLF [cardiac death, target vessel-related myocardial infarction (TV-MI), or ischaemia-driven target lesion revascularization (ID-TLR)], target vessel failure [TVF; cardiac death, MI, or ischaemiadriven target vessel revascularization (ID-TVR)], and major adverse cardiac events (MACE; cardiac death, MI, or IDTLR), along with the individual component endpoints of these endpoints and device thrombosis. Device thrombosis was defined as acute ( $<24$ hours), subacute (1-30 days), late (30 days -1 year), and very late (beyond 1 year), and the level of evidence (definite or probable) was determined according 
Table 1 Baseline demographic and procedural information of the intention-to-treat population

\begin{tabular}{|c|c|}
\hline Parameters & $\mathrm{N}=30$ \\
\hline Age (years) & $53.5 \pm 9.9$ \\
\hline Male (\%) & $21(70.0)$ \\
\hline BMI $\left(\mathrm{kg} / \mathrm{m}^{2}\right)$ & $24.8 \pm 2.9$ \\
\hline Current smoker (\%) & $7(23.3)$ \\
\hline Diabetes (\%) & $4(13.3)$ \\
\hline Hypertension (\%) & $16(53.3)$ \\
\hline Hyperlipidemia (\%) & $4(13.3)$ \\
\hline Previous MI (\%) & $2(6.7)$ \\
\hline Stable angina (\%) & $5(16.7)$ \\
\hline Unstable angina (\%) & $23(76.6)$ \\
\hline Left ventricular ejection fraction (\%) & $64.2 \pm 4.2$ \\
\hline Lesion number & 30 \\
\hline \multicolumn{2}{|l|}{ ACC/AHA lesion type } \\
\hline$A(\%)$ & $30(100.0)$ \\
\hline B1/B2/C (\%) & 0 \\
\hline \multicolumn{2}{|l|}{ Target vessel } \\
\hline LAD (\%) & $16(53.3)$ \\
\hline LCX (\%) & $8(26.7)$ \\
\hline $\mathrm{RCA}(\%)$ & $6(20.0)$ \\
\hline
\end{tabular}

Table 1 (continued)

to the Academic Research Consortium definitions (7). All clinical events were monitored by an independent clinical events committee.

\section{Statistical analysis}

All analyses were performed according to the intention-totreat (ITT) principle. Continuous variables are presented as the mean \pm SD. Normally distributed continuous variables were compared by Student's $t$-test. All statistical analyses were two-sided and performed with the significance level set at 0.05. SAS software, version 9.4 (SAS Institute, Cary, North Carolina) was used to perform all statistical analyses.
Table 1 (continued)

\begin{tabular}{|c|c|}
\hline Parameters & $\mathrm{N}=30$ \\
\hline Previous target vessel intervention & 0 \\
\hline Mean diameter of reference vessel (mm) & $2.90 \pm 0.30$ \\
\hline Minimal luminal diameter (mm) & $1.20 \pm 0.40$ \\
\hline Diameter stenosis (\%) & $58.9 \pm 12.4$ \\
\hline Lesion length (mm) & $12.75 \pm 3.84$ \\
\hline Eccentric (\%) & $23(76.7)$ \\
\hline Pre-dilation performed (\%) & $30(100.0)$ \\
\hline Diameter of balloon for pre-dilation (mm) & $2.80 \pm 0.23$ \\
\hline Maximum pressure for pre-dilation (atm) & $9.9 \pm 2.1$ \\
\hline Post-dilation performed (\%) & $27(90.0)$ \\
\hline Diameter of balloon for post-dilation (mm) & $3.20 \pm 0.16$ \\
\hline Maximum pressure for post-dilation (atm) & $16.4 \pm 3.7$ \\
\hline Number of study devices implanted & 30 \\
\hline Number of bailout stents implanted & 1 \\
\hline Acute luminal gain (mm) & $1.43 \pm 0.43$ \\
\hline Bailout stenting (\%) & $1(3.3)$ \\
\hline Total contrast use (mL) & $145.7 \pm 36.0$ \\
\hline Device success (\%) & $30(100.0)$ \\
\hline \multicolumn{2}{|c|}{$\begin{array}{l}\text { ITT, intention-to-treat; BMI, body mass index; MI, myocardial } \\
\text { infarction; ACC, American College of Cardiology; AHA, American } \\
\text { Heart Association; LAD, left anterior descending artery; LCX, left } \\
\text { circumflex artery; RCA, right coronary artery. }\end{array}$} \\
\hline
\end{tabular}

\section{Results}

\section{Patients and baseline characteristics}

Thirty eligible patients in two centres were enrolled in this study between September 2013 and January 2014, there were 19 patients from Zhongshan Hospital Fudan University and 11 patients from 301 Hospital. Informed consent was obtained from all patients before participation in the study. The patients' baseline demographic and procedural information is shown in Table 1. Each of the 30 study devices was implanted successfully. Only one patient received bailout stenting due to distal edge dissection. All lesions were classified as ACC/AHA lesion classification 


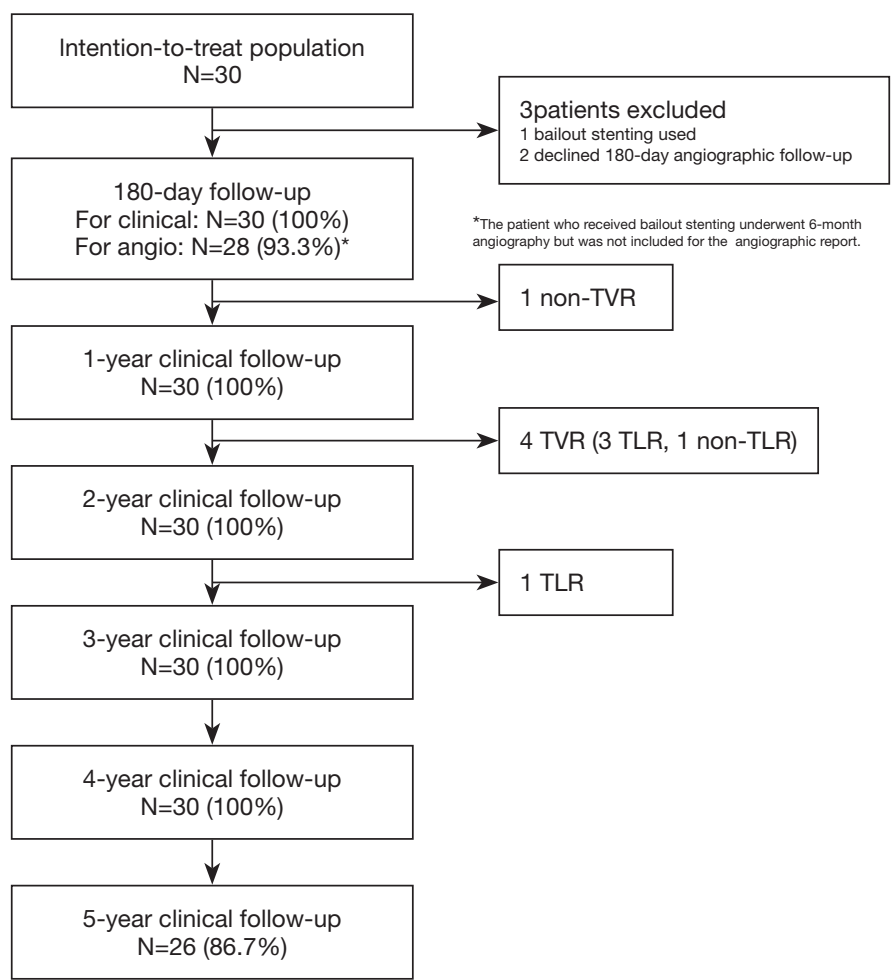

Figure 1 The flow chart of the XINSORB first-in-human study. TVR, target vessel revascularization; TLR, target lesion revascularization.

type A. More than $50 \%$ of the lesions were located on the left anterior descending branch. All lesions were predilated. The majority of the lesions were postdilated with a noncompliance balloon at a high pressure. All patients were discharged with no clinical events.

\section{Long-term follow-up and clinical outcomes}

Figure 1 shows the patient flow chart of this study. Two patients declined invasive follow-up at 6 months. The patient who received bailout stenting underwent angiography at 6 months but was not included in the angiographic report. Consequently, the per-treatmentevaluation population comprised 27 patients, all of whom were clinically followed for 4 years. At 5 years, 4 patients were lost to follow-up after not answering the phone calls.

At 337 days after the index procedure, 1 patient suffered nontarget vessel $\mathrm{Q}$ wave myocardial infarction and received additional intervention. Four patients underwent TVR at the 2-year follow-up, including 3 patients with ID-TLR. Two of these patients experienced recurrent chest pain at 612 and 665 days. Repeat angiography showed restenosis at the
XINSORB scaffold-treated sites, and metallic stents were implanted. The third patient experienced chest pain onset leading to non-Q-wave myocardial infarction 530 days after the index procedure. An electrocardiogram after admission to the hospital showed ST-segment elevation. An emergent angiogram was carried out immediately, revealing extensive in-scaffold thrombosis with a TIMI flow of grade 3. Optical coherent tomography (OCT) revealed scaffold discontinuity with white thrombosis. The treatment involved the placement of a metallic stent to cover the implanted scaffold. (Figure 2) At the 3-year follow-up, 1 more case of ID-TLR was recorded. This patient presented with chest discomfort and was admitted to the hospital 835 days after the index procedure. Restenosis was discovered, and reintervention was carried out. All 5 cases of TVF were revascularized by percutaneous coronary intervention (PCI). The follow-up continued, and no more clinical events were reported from 3-5 years. No cardiac deaths occurred during observation. Table 2 contains detailed information on the clinical outcomes. Figure 3 showed the Kaplan-Meier survival curve of TLF, MACE, scaffold thrombosis, and allcause death through 5 years follow-up in this FIM study. 

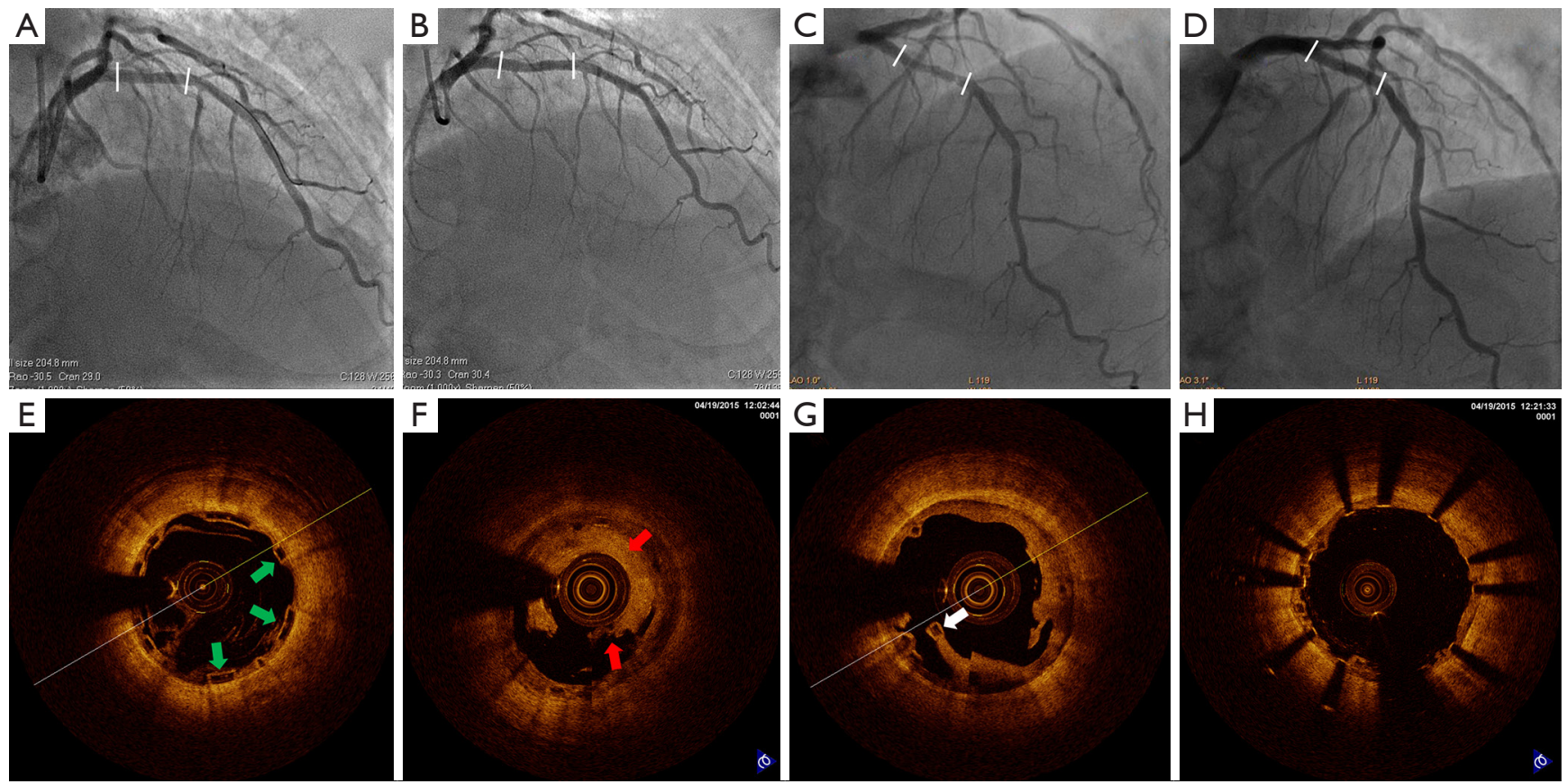

Figure 2 The patient with very late confirmed scaffold thrombosis. Favorable post-procedural angiographic result (A) and 6 months angiographic and OCT findings (B,E). In-scaffold thrombosis was revealed 530 days after the index procedure (C). OCT showed scaffold discontinuity $(\mathrm{G})$ with white thrombosis $(\mathrm{F})$. A metallic stent was implanted (D) with good OCT imagings (H).

\section{Device thrombosis}

Only one very late, confirmed case of thrombosis was recorded at the 5 -year follow-up (at exactly 530 days) in this study. This case is described in detail in the text above. No further events related to thrombosis were recorded from $2-5$ years.

\section{Typical case}

Twenty-eight patients routinely also received a 6-month invasive follow-up protocol, and 3 patients underwent an additional voluntary angiographic examination at 2 or 3 and 5 years without selection. No clinical symptoms were experienced by these patients. We previously reported 5 years of serial intravascular imaging outcomes of XINSORB BRS (8). This report was the first XINSORB FIM study. The first case was a 35-year-old man with limited risk factors for atherosclerotic heart disease. Severe narrowing of the distal right coronary artery was revealed by angiography. After predilation, a $3.0 \times 18 \mathrm{~mm}$ XINSORB scaffold was deployed. Repeat angiogram showed no residual stenosis and grade 3 TIMI flow. The patient was admitted for angiographic follow-up at 6 months and at 2,3 , and 5 years after the index procedure. Angiography showed a widely patent lumen at the treated site. Over time, strut "boxes" become progressively smaller and less clear under OCT, reflecting material resorption. Some "black boxes" could still be seen at the 5 -year follow-up, although most struts were absorbed. The second case was a 51-year-old man with hypertension. Angiography detected severe stenosis at the middle of the circumflex artery. The patient was treated with a XINSORB scaffold measuring $3.0 \times 18 \mathrm{~mm}$. The lumen remained patent for over 5 years, as shown by angiograms. OCT showed that all struts had completely disappeared at the 5-year follow-up. The final case was a 45-year-old man with hypertension and diabetes mellitus, who was also a smoker. Angiography showed a single lesion proximal to the left anterior descending artery. A XINSORB scaffold of $3.0 \times 15 \mathrm{~mm}$ was implanted. The results were similar to those in the previous case. The struts were completely absorbed, with a patent lumen. Figure 4 shows the angiograms and OCT images of cases 2 and 3. 
Table 2 The 5 -year clinical outcomes of the intention-to-treat population $(\mathrm{N}=30)$

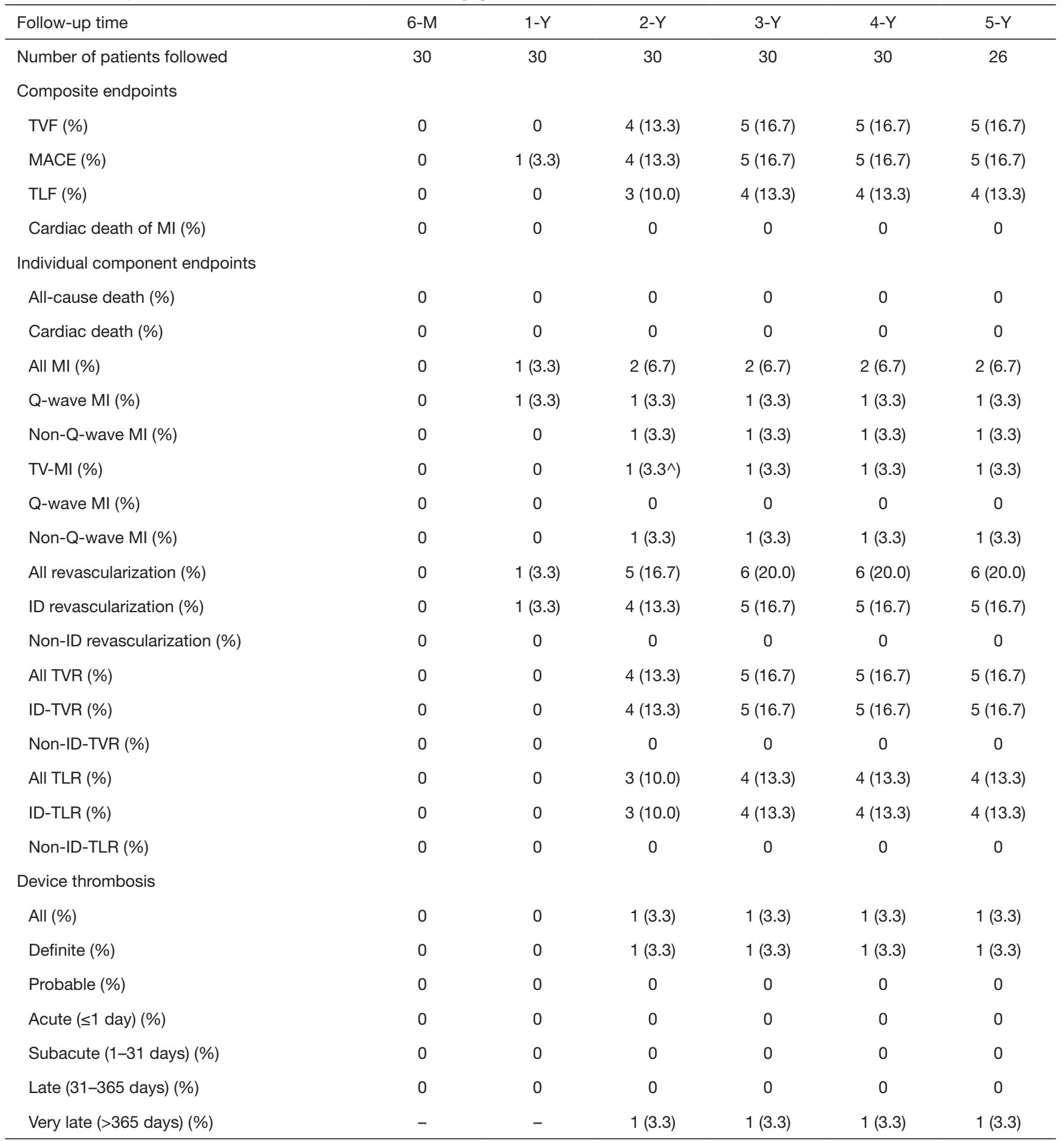

TVF, target vessel failure; MACE, major adverse cardiac event; TLF, target lesion failure; MI, myocardial infarction; TV-MI, target vessel-related myocardial infarction; ID, ischaemia-driven; TVR, target vessel revascularization; TLR, target lesion revascularization. 

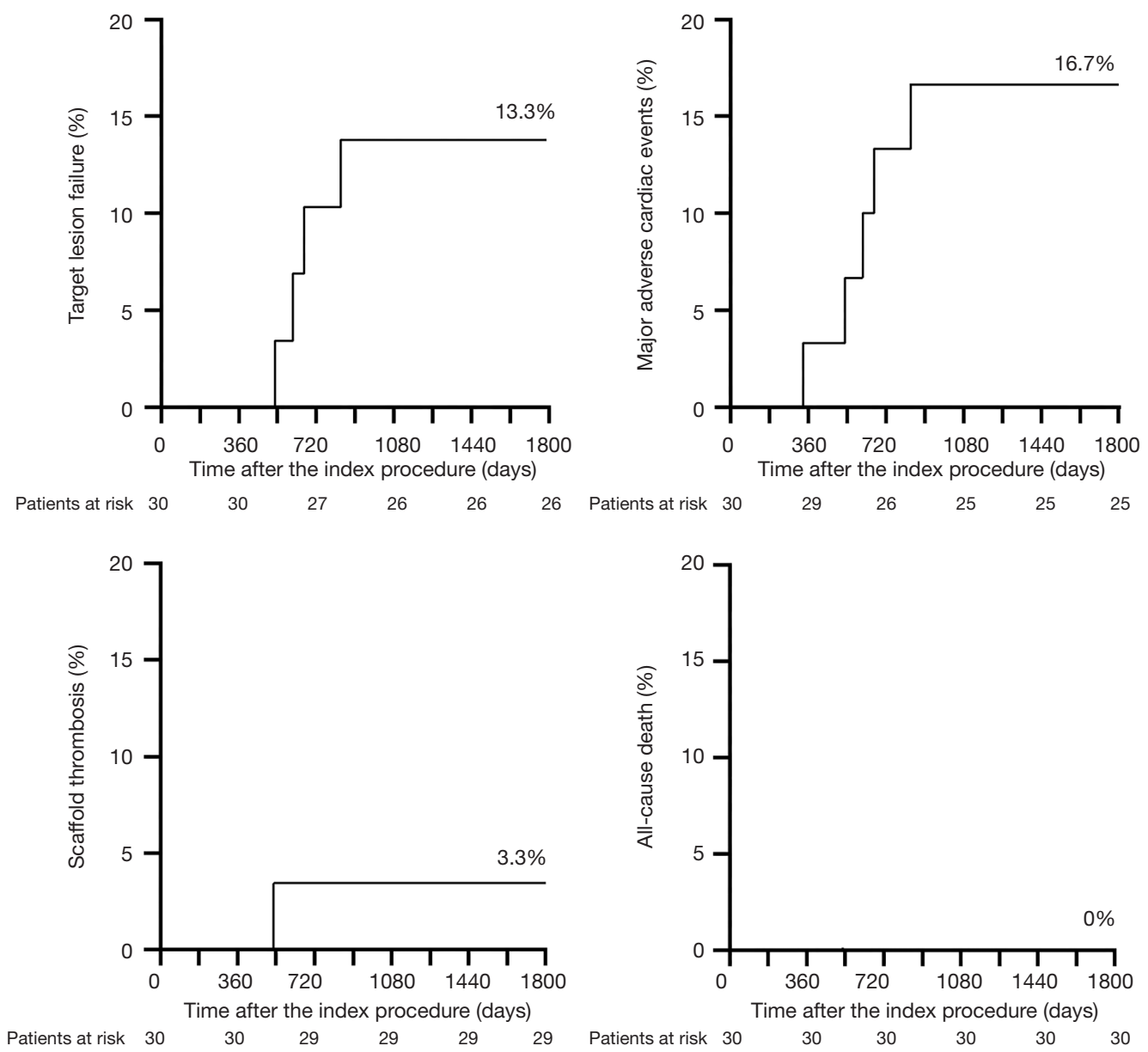

Figure 3 The Kaplan-Meier survival curve of TLF, MACE, scaffold thrombosis, and all-cause death through 5 years follow-up in this FIM study. TLF, target lesion failure; MACE, major adverse cardiac events; FIM, first-in-human.

\section{Discussion}

The main findings of this study were as follows: (I) considering that this FIM study was launched at an early stage of the BRS era without optimal implantation techniques, the clinical outcomes including TLF, MACE, and ID-TLR in the 5-year follow-up were acceptable; (II) no clinical events were recorded beyond 3 years; and (III) the rate of thrombosis was relatively low.

The long-term results up to 3 years showed that the rates of TLF and device thrombosis with Absorb BRS were significantly higher than those with the everolimus-eluting stent (EES) $(1,2)$. The 3-year outcomes of the ABSORB II study showed that the rates of TLF and device thrombosis were $10 \%$ and $3 \%$ with Absorb BRS, respectively; these rates were significantly higher than those with the EES (5\% and $0 \%$, respectively). Similarly, the 3-year results from the ABSORB III study demonstrated that the rate of device thrombosis with Absorb BRS was significantly greater than that with the EES ( $2.3 \%$ vs. $0.7 \%, \mathrm{P}=0.01)$, although the rate of TLF was comparable between the two devices (13.4\% vs. $10.4 \%, \mathrm{P}=0.055)$. However, Stone et al. indicated that the period of excess risk for the first generation of BRSs might end after 3 years. In a recently published meta-analysis (4), TLF occurred in $11.6 \%$ of the Absorb BRS-treated patients vs. $7.9 \%$ of the EES-treated patients between $0-3$ years (HR, 1.42; 95\% CI, 1.12-1.80), and in $4.3 \%$ of the BVStreated patients vs. $4.5 \%$ of the EES-treated patients between 3-5 years (HR, 0.92; 95\% CI, 0.64-1.31). Device thrombosis occurred in $2.4 \%$ of the Absorb BRS-treated patients $v$ s. $0.6 \%$ of the EES-treated patients between $0-3$ years (HR, 3.86; 95\% CI, 1.75-8.50) and $0.1 \%$ of the 

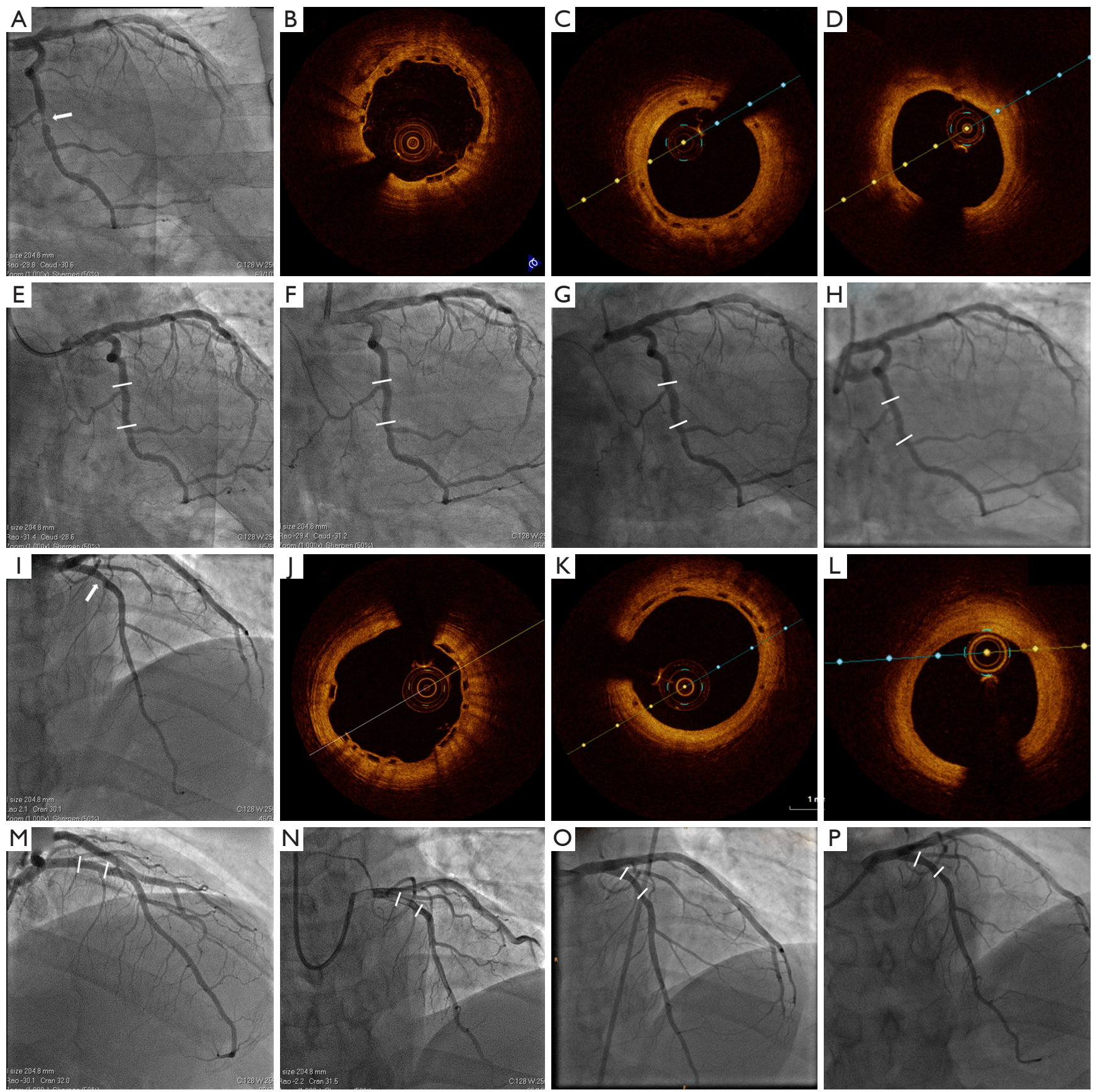

Figure 4 Long-term results of two typical cases in the XINSORB FIM study. One was a 51-year-old man with severe stenosis at the middle of the circumflex artery (A, white arrow). A XINSORB scaffold measuring 3.0×18 mm was implanted (E). The lumen remained patent for over 5 years $(\mathrm{F}, \mathrm{G}, \mathrm{H})$. OCT showed that all struts gradually absorbed and completely disappeared at the 5-year follow-up (B,C,D). The other case was a 45-year-old man with a single lesion proximal to the left anterior descending artery (I, white arrow). A XINSORB scaffold of $3.0 \times 15 \mathrm{~mm}$ was deployed (M). The struts were completely absorbed (J,K,L), with a patent lumen (N,O,P). Between the white lines was the implanted scaffolds. FIM, first-in-human; OCT, optical coherent tomography. 
BVS-treated patients vs. $0.3 \%$ of the EES-treated patients between 3-5 years (HR, 0.44; 95\% CI, 0.07-2.70). After 3 years, the results slightly favoured Absorb BRS, but there was no significant difference.

The results of this FIM study were similar to those of pooled ABSORB serial studies. Before 3 years, the rates of TLF and thrombosis were clearly increased among the BRS-treated patients; however, after 3 years, the rates of these adverse events remained steady. The results from this FIM study constitute new evidence that supports the favourable long-term outcomes of BRSs after the 3-year follow-up. The XINSORB FIM study was initiated at the early stage of the BRS era. Technologies such as intravascular imaging and optimal implantation were not utilized in the study; however, they are now considered standard processes in today's practice of BRS implantation. During the 3-year follow-up, the rates of TLF and thrombosis were slightly increased. Most device failures were attributable to restenosis, leading to ischaemia and revascularization. It is possible that it took up to 3 years for the first generation of BRSs to be fully absorbed. After complete absorption, the natural vessel wall was restored, with limited inflammation $(9,10)$. The rates of device failure and clinical events could continue to increase before the vessel wall was completely repaired. The results from the meta-analysis and our study matched those obtained from the preclinical study. Vessel wall inflammation and repair play pivotal roles in clinical outcomes.

Most adverse events were attributed to the relatively thick struts of the first generation of BRSs, which required adequate mechanical properties. The optimal device implantation technique was also central to obtaining acute and long-term outcomes. However, during the absorption process of BRSs, a unique failure mode known as intraluminal scaffold dismantling can occur (11-13). In our study, the case of thrombosis was caused by strut discontinuity or fracture, which was one of the negative consequences of bulk erosion. Another failure mode of BRSs is neoatherosclerosis (14). In this study, we reviewed 4 cases of TLR. Two of these cases suffered ischaemic symptoms from restenosis. OCT showed neoatherosclerosis at the previously stented site. However, the mechanism of neoatherosclerosis in BRS is still unknown.

After the implantation of coronary devices, maintaining drug therapy, including statins and anti-platelet agents, for a lifetime is crucial to inhibiting neointima hyperplasia and reducing thrombosis. However, it is unknown for how long dual antiplatelet therapy (DAPT) should be administered to patients with BRSs. Thicker struts have a clearly thrombogenic effect (15). Furthermore, the PLLA nature of the BRS limits sizing and implantation technology to prevent fracture, underexpansion, or incomplete apposition, which might lead to delayed healing or floating struts into the lumen (16-18). The current recommendation suggests that the duration of DAPT treatment should be prolonged until the BRS is completely resorbed (19). In a much earlier XINSORB FIM study, patients were prescribed aspirin and clopidogrel for at least 12 months. However, in later RCTs of XINSORB, the duration of DAPT was modified. In fact, $59 \%$ of the XINSORB-treated patients in the XINSORB RCT were still on DAPT at the 3-year follow-up.

Along with BRS came a concept called vascular restoration therapy (VRT). BRS supports the vessel wall, resisting acute occlusion after implantation until negative remodeling is terminated. It can be resorbed and, subsequently, the vessel can be released from the perpetual caging of metallic stents. However, VRT is a complex process in which safe BRS implantation should be the first step. A systemic treatment targeted to the underlying chronic disease would be necessary to ameliorate omnipresent endothelial dysfunction demonstrated in patients with coronary risk factors and atherosclerosis.

This study has some limitations. First, the sample size was extremely small. Only 30 patients were enrolled in this FIM study. However, this study was designed to investigate the preliminary safety and efficacy of XINSORB BRS. According to the guidelines of the CFDA, 30 patients constitutes a reasonable sample size, and no control group was needed. Second, this study did not use intravascular imaging. The importance of invasive imaging modalities, from angiography to sound- and light-based techniques, in guiding BRS implantation procedures and assessing the acute results post implantation as well as the long-term changes until complete resorption, has been proven (20). However, the importance of these imaging techniques was not emphasized at the beginning of the BRS era. Early studies, including this study and the ABSORB China study, neither recommended nor encouraged these methods, even in the protocols. Third, optimal implantation techniques, such as the PSP technique (proper lesion preparation, vessel sizing, and postdilation with high pressure), are recommended to improve the long-term outcomes of BRS implantation; however, these techniques were not used in this FIM study (19). Although the rates of predilation and postdilation were $100 \%$ and $90 \%$, respectively, the diameter of the balloon was $2.5 \mathrm{~mm}$ in most cases of predilation. 
Visualization was used for sizing instead of QCA or other intravascular imaging techniques. The study design also had limitations. Fourth, the lesions treated in this FIM study were fairly simple. The experience acquired from this study could not be extrapolated to more complex lesions. Finally, the scaffold used in this study was a firstgeneration BRS, which meant thicker struts, a large profile, low deliverability, and poor compliance with high risks of restenosis and thrombosis. The next generation of BRSs with favourable expansion properties and minimal recoil has been developed. Improved 3-year results with a $100 \mu \mathrm{m}$ PLLA-based BRS have been reported (21). If newly designed BRSs can achieve comparable results to those of traditional metallic stents during the active bioresorption phase, BRS technology might be a suitable option for many patients with coronary artery disease.

\section{Conclusions}

Considering that this FIM study was launched at an early stage of the BRS era and did not use optimal implantation techniques, the clinical outcomes of TLF during the 5-year follow-up were acceptable. The rate of thrombosis was relatively low.

\section{Acknowledgments}

Funding: This work was funded by grants from the National Natural Science Foundation of China (Nos. 81521001 and 81670319) and the National Key Research and Development Program (Nos. 2016YFC1102300 and 2016YFC1102303).

\section{Footnote}

Reporting Checklist: The authors have completed the TREND reporting checklist. Available at http://dx.doi. org/10.21037/atm-20-5668

Data Sharing Statement: Available at http://dx.doi. org/10.21037/atm-20-5668

Conflicts of Interest: All authors have completed the ICMJE uniform disclosure form (available at http://dx.doi. org/10.21037/atm-20-5668). The authors have no conflicts of interest to declare.

Ethical Statement: The authors are accountable for all aspects of the work in ensuring that questions related to the accuracy or integrity of any part of the work are appropriately investigated and resolved. All procedures performed in this study involving human participants were in accordance with the Declaration of Helsinki (as revised in 2013). The study was approved by institutional ethics committee of Zhongshan Hospital, Fudan University (No. 2013-36) and informed consent was taken from all the patients.

Open Access Statement: This is an Open Access article distributed in accordance with the Creative Commons Attribution-NonCommercial-NoDerivs 4.0 International License (CC BY-NC-ND 4.0), which permits the noncommercial replication and distribution of the article with the strict proviso that no changes or edits are made and the original work is properly cited (including links to both the formal publication through the relevant DOI and the license). See: https://creativecommons.org/licenses/by-nc-nd/4.0/.

\section{References}

1. Serruys PW, Chevalier B, Sotomi Y, et al. Comparison of an everolimus-eluting bioresorbable scaffold with an everolimus-eluting metallic stent for the treatment of coronary artery stenosis (ABSORB II): a 3 year, randomised, controlled, single-blind, multicentre clinical trial. Lancet 2016;388:2479-91.

2. Kereiakes DJ, Ellis SG, Metzger C, et al. 3-Year Clinical Outcomes With Everolimus-Eluting Bioresorbable Coronary Scaffolds: The ABSORB III Trial. J Am Coll Cardiol 2017;70:2852-62.

3. Tijssen RYG, Kraak RP, Hofma SH, et al. Complete two-year follow-up with formal non-inferiority testing on primary outcomes of the AIDA trial comparing the Absorb bioresorbable scaffold with the XIENCE drugeluting metallic stent in routine PCI. EuroIntervention 2018;14:e426-33.

4. Stone GW, Kimura T, Gao R, et al. Time-Varying Outcomes With the Absorb Bioresorbable Vascular Scaffold During 5-Year Follow-up: A Systematic Metaanalysis and Individual Patient Data Pooled Study. JAMA Cardiol 2019;4:1261-9.

5. Wu Y, Shen L, Ge L, et al. Six-month outcomes of the XINSORB bioresorbable sirolimus-eluting scaffold in treating single de novo lesions in human coronary artery. Catheter Cardiovasc Interv 2016;87 Suppl 1:630-7.

6. Wu Y, Shen L, Yin J, et al. Twelve-month angiographic 
and clinical outcomes of the XINSORB bioresorbable sirolimus-eluting scaffold and a metallic stent in patients with coronary artery disease. Int J Cardiol 2019;293:61-6.

7. Cutlip DE, Windecker S, Mehran R, et al. Clinical end points in coronary stent trials: a case for standardized definitions. Circulation 2007;115:2344-51.

8. Wu Y, Shen L, Yin J, et al. 5 Years of Serial Intravascular Imaging Outcomes of XINSORB Sirolimus-Eluting Bioresorbable Vascular Scaffold. JACC Cardiovasc Interv 2019;12:602-3.

9. Cheng Y, Gasior P, Xia JG, et al. Comparative Biomechanical Behavior and Healing Profile of a Novel Thinned Wall Ultrahigh Molecular Weight Amorphous Poly-1-Lactic Acid Sirolimus-Eluting Bioresorbable Coronary Scaffold. Circ Cardiovasc Interv 2017;10:e005116.

10. Nakatani S, Ishibashi Y, Sotomi Y, et al. Bioresorption and Vessel Wall Integration of a Fully Bioresorbable Polymeric Everolimus-Eluting Scaffold: Optical Coherence Tomography, Intravascular Ultrasound, and Histological Study in a Porcine Model With 4-Year Follow-Up. JACC Cardiovasc Interv 2016;9:838-51.

11. Kereiakes DJ, Onuma Y, Serruys PW, et al. Bioresorbable vascular scaffolds for coronary revascularization. Circulation 2016;134:168-82.

12. Stone GW, Abizaid A, Onuma Y, et al. Effect of technique on outcomes following bioresorbable vascular scaffold implantation: analysis from the ABSORB trials. J Am Coll Cardiol 2017;70:2863-74.

13. Stone GW, Granada JF. Very late thrombosis after bioresorbable scaffolds: cause for concern? J Am Coll Cardiol 2015;66:1915-7.

14. Moriyama N, Shishido K, Tanaka Y, et al. Neoatherosclerosis 5 Years After Bioresorbable Vascular

Cite this article as: Wu Y, Yin J, Chen J, Yao Z, Qian J, Shen L, Ge L, Ge J; on behalf of the XINSORB first-in-human study. Final report of the 5 -year clinical outcomes of the XINSORB bioresorbable sirolimus-eluting scaffold in the treatment of single de novo coronary lesions in a first-in-human study. Ann Transl Med 2020;8(18):1162. doi: 10.21037/atm-20-5668
Scaffold Implantation. J Am Coll Cardiol 2018;71:1882-93.

15. Kang SH, Chae IH, Park JJ, et al. Stent Thrombosis With Drug-Eluting Stents and Bioresorbable Scaffolds: Evidence From a Network Meta-Analysis of 147 Trials. JACC Cardiovasc Interv 2016;9:1203-12.

16. Tamburino C, Latib A, van Geuns RJ, et al. Contemporary practice and technical aspects in coronary intervention with bioresorbable scaffolds: a European perspective. EuroIntervention 2015;11:45-52.

17. Gomez-Lara J, Radu M, Brugaletta S, et al. Serial analysis of the malapposed and uncovered struts of the new generation of everolimus-eluting bioresorbable scaffold with optical coherence tomography. JACC Cardiovasc Interv 2011;4:992-1001.

18. Räber L, Brugaletta S, Yamaji K, et al. Very late scaffold thrombosis: intracoronary imaging and histopathological and spectroscopic findings. J Am Coll Cardiol 2015;66:1901-14.

19. Byrne RA, Stefanini GG, Capodanno D, et al. Report of an ESC-EAPCI Task Force on the evaluation and use of bioresorbable scaffolds for percutaneous coronary intervention: executive summary. Eur Heart J 2018;39:1591-601.

20. Chamié D, Garcia-Garcia H, Costa RA, et al. Role of invasive imaging in acute and long-term assessment of bioresorbable scaffold technology. Catheter Cardiovasc Interv 2016;88:38-53.

21. Seth A, Onuma Y, Chandra P, et al. Three-year clinical and two-year multimodality imaging outcomes of a thinstrut sirolimus-eluting bioresorbable vascular scaffold: MeRes-1 trial. EuroIntervention 2019;15:607-14.

(English Language Editor: J. Reynolds) 